
\title{
$\begin{array}{ll}\text { Research Square } & \text { Preprints are preliminary reports that have not undergone peer review. } \\ \text { They should not be considered conclusive, used to inform clinical practice, } & \text { or referenced by the media as validated information. }\end{array}$
}

\section{A New Bioreactor to Promote Follicular Growth With or Without Activin A}

\section{Cynthia Jovet}

Hôpital Femme Mère Enfant: Hopital Femme Mere Enfant

\section{Eloise Fraison ( $\sim$ fraison.eloise@yahoo.fr)}

HFME: Hopital Femme Mere Enfant https://orcid.org/0000-0001-8083-1346

\section{Jacqueline Lornage}

Hôpital Femme Mère Enfant: Hopital Femme Mere Enfant

Nicolas Morel-journel

Groupement Hospitalier Édouard Herriot: Groupement Hospitalier Edouard Herriot

\section{Antoine Gavoille}

GH Edouart Herriot: Groupement Hospitalier Edouard Herriot

\section{David Laurent}

Claude-Bernard University: Universite Claude Bernard Lyon 1

\section{Alexandra Montembault}

UCBL 1: Universite Claude Bernard Lyon 1

\section{Cyrielle Fournier}

Université Lyon 1 Faculte de Médecine Lyon-Est: Universite Lyon 1 Faculte de Medecine Lyon-Est

\section{Bruno Salle}

Hôpital Femme Mère Enfant: Hopital Femme Mere Enfant

\section{Elsa Labrune}

Hôpital Femme Mère Enfant: Hopital Femme Mere Enfant

\section{Research}

Keywords: In vitro folliculogenesis, bioreactor, Activin A, follicular growth

Posted Date: September 20th, 2021

DOI: https://doi.org/10.21203/rs.3.rs-903355/v1

License: (9) This work is licensed under a Creative Commons Attribution 4.0 International License. Read Full License 


\section{Abstract}

Background: The aim of the present study was to evaluate the effect of Activin A on the activation of in vitro folliculogenesis of human ovarian tissues with or without our new three-dimensional structure (3Dstructure).

Methods: Five fresh ovarian human tissues were cultured in vitro in 4 groups with $100 \mathrm{ng} / \mathrm{mL}$ Activin A or without Activine A and within or without a 3D-structure for 20 or 22 days of in vitro culture. Follicular density and quality were evaluated, and follicular diameters were measured. Estradiol secretion was quantified. Proliferation and apoptosis through immunostaining were performed.

Results: The proportion of primordial follicles was significantly reduced, and the proportion of primary and secondary follicles was significantly increased in all four groups $(p<0.001)$. Antral cavities were observed in the four culture groups. Activin A supplementation did not significantly affect the follicular density, follicular quality, follicular growth, or estradiol secretion ( $p>0.05$ ). The 3D-structure increased the density of primary follicles and decreased the estradiol secretion $(p<0.001)$. Tissular proliferation was significantly lower in the 3D-structure group compared to the non-3D-structure group $(p=0.008)$. Regarding tissular apoptosis, it was significantly higher in the Activin group compared to the non-Activin group (0.006).

Conclusion: The presence of Activin A did not seem to play a key role in in vitro folliculogenesis activation. However, the results may indicate that the 3D-structure could be more physiological and could prevent a pejorative in vitro folliculogenesis flare-up.

\section{Background}

Ovarian tissue cryopreservation and autograft is the only option available for prepubertal girls and patients who cannot delay their cancer treatment. However, in acute leukemia for example, there is a risk of reintroducing malignant cells with the ovarian tissue transplantation (1). Therefore, in vitro systems have been developed to support the growth and maturation of follicles, a fortiori oocytes, from immature to final stage. Metaphase II oocytes have been obtained, thanks to a multi-step culture system using Activin A, opening the possibility of in vitro fertilization as an option for women who cannot use their own ovarian tissue (2).

Several culture systems are currently used to support in vitro folliculogenesis: Matrigel ${ }^{\circledR}$ (BD Bioscience, San Jose, CA), gelatin structure, alginate drop, or synthetic extracellular matrix like components. Most culture systems are currently based on enzymatic or mechanical isolation of follicles $(3,4,5,6,7)$. An alternative approach is to work on ovarian fragments rather than on an isolated follicle, in order to maintain the architecture and interactions of the follicles. The use of Activin A combined with a proper culture system is reported to activate the primordial follicles and to initiate follicle growth. Activin A is a dimeric glycoprotein belonging to the transforming growth factor beta superfamily and its receptors are expressed in primordial, primary, and secondary follicles. It stimulates the granulosa cell proliferation, 
follicle, and oocyte growth. In addition to this, it also accelerates oocyte maturation and stimulates aromatase $(8,9)$. Our team is working on an innovative 3D-structure made from chitosan, which can maintain the integrity of the ovarian tissue. The ovarian fragment is placed into a bioreactor, which is sealed on each end. This encapsuled system enables the paracrine factors to be maintained within the ovarian tissue.

The aim of this study was, therefore, to evaluate the activation of in vitro folliculogenesis with or without Activin A, within or without our new 3D-structure of human ovarian tissue. We compared four culture groups for a 20- or 22-days in vitro culture based on the presence or absence of Activin A and on the presence or absence of a 3D-structure.

\section{Methods}

\section{Ovarian tissue}

Fresh ovaries $(n=5)$ were obtained with informed consent from women undergoing a sex reassignment surgery from female to male gender (Centre Hospitalier Lyon Sud, urology department). Mean age of the patients was $25 \pm 7$ years. They all received a treatment by testosterone with a mean duration of $25.3 \pm 18.4$ months. Approval of this study was given by the local ethics committee. Ovarian tissue was transported to the laboratory in dissection medium (Minimum Essential Medium, Alpha 1X, Corning Life Sciences, NY, USA) within 30 minutes at the temperature of $4-10^{\circ} \mathrm{C}$.

\section{Bioreactor}

The new 3D-structure was a tube-shaped hydrogel made from chitosan, license number FR1910681. Chitosan hydrogel bioreactors were prepared as described in 2008 by David et al. (10) and Araizi et al. (11). Chitosan was first purified as previously described by Montembault et al. (12) then chitosan hydrogels were prepared by gelation of aqueous chitosan acetate solutions. Briefly, purified chitosan was dissolved in an acetic acid solution at a polymer concentration of $0.5 \%$ then filtered successively through 5, 3, 0.8 and $0.42 \mu \mathrm{m}$ filters (Millipore, Molsheim, France). Filtered chitosan acetate was next precipitated with ammonia, washed with deionized water, and freeze-dried. Later, a chitosan solution at $2 \% \mathrm{w} / \mathrm{w}$ polymer concentration was obtained by dispersion of neutralized chitosan lyophilizate in water plus acetic acid added in stoichiometric amounts sufficient to achieve protonation of $-\mathrm{NH} 2$ groups of glucosamine residues. That solution was centrifuged, aspired in dispenser syringes (Nordson EFD, Westlake, $\mathrm{OH}$ ) and extruded through a 6-mm diameter tip using Performus I dispenser (Nordson EFD). The extrudate was neutralized for $2 \mathrm{~min}$ in sodium hydroxide $1 \mathrm{M}$ to coagulate the external part of the extrudate. This resulted in a tubular hydrogel that was stabilized in deionized water. The uncoagulated inner solution was removed by an airflow creating a hollow tube. These 4-mm diameter and 1-mm membrane 'bioreactors' were sterilized for $20 \mathrm{~min}$ at $121^{\circ} \mathrm{C}$ in an autoclave and stored until use in deionized water at room temperature. The ovarian tissue fragments were placed into the bioreactor and the tube was sealed with suture wire on each end. 


\section{Tissue preparation and ovarian fragments culture}

Following transport, the ovarian tissue was transferred to a fresh dissection medium (Minimum Essential Medium, Alpha 1X, Corning Life Sciences, NY, USA) and then cut lengthwise in two fragments. For each fragment, the medulla was removed using flat dissection scissors, thus obtaining two hemi cortexes. These hemi cortexes were dissected into ovarian fragments of $\sim 3 \times 3 \times 1 \mathrm{~mm}$.

One fresh fragment was taken and fixed in $4 \%$ paraformaldehyde at room temperature during $24 \mathrm{~h}$ for histological evaluation whereas the remaining fragments were placed randomly in four culture groups (Figure 1):

Group A: ovarian fragment placed in culture medium without Activin A without 3D-structure

Group B: ovarian fragment placed in culture medium with Activin A but without 3Dstructure

Group C: ovarian fragment placed in culture medium without Activin A but within a 3D-structure

Group D: ovarian fragment placed in culture medium with Activin A within 3D-structure

Activin A (A4941-10UG, Sigma-Aldrich, Saint Louis, USA) was used at the dose of 100 $\mathrm{ng} / \mathrm{ml}$, according to literature.

Ovarian fragments with any visible antral follicles under light microscopy were not included.

8 fragments (of $\sim 3 \times 3 \times 1 \mathrm{~mm}$ ) from each patient $(n=5)$ were cultured in $A, B, C$ and $D$ groups (2 fragments per group). Thus, a total of 40 fragments were incubated individually in cell culture plates containing $3 \mathrm{~mL}$ of culture MEM Alpha Medium (Corning LifeSciences, NY, USA) supplemented with Lglutamine, $10 \%$ of serum substitute supplement (SSS; Irvine scientific, Santa Ana, USA), $100 \mathrm{UI} / \mathrm{mL}$ of penicillin $\mathrm{G}$ and $100 \mu \mathrm{g} / \mathrm{mL}$ of streptomycin (HyClone, South Logan, USA), $2.5 \mu \mathrm{g} / \mathrm{mL}$ of amphotericin B (PAN-Biotech, Bayern, Germany), $10 \mathrm{ng} / \mathrm{mL}$ of insulin, $10 \mathrm{ng} / \mathrm{mL}$ of transferrin, $10-3 \mu \mathrm{g} / \mathrm{mL}$ of selenium (PAN-Biotech), $25 \mathrm{mUl} / \mathrm{mL}$ of rFSH (Bemfola ${ }^{\circledR}$, Gedeon Richter, Budapest, Hungary), $50 \mu \mathrm{g} / \mathrm{mL}$ of ascorbic acid (Sigma-Aldrich, St Quentin Fallavier, France) and $1 \mathrm{~g} / \mathrm{L}$ of glucose (Sigma-Aldrich, St Quentin Fallavier, France).

Ovarian fragments assigned to groups A and B were placed in culture wells, whereas ovarian fragments assigned to groups $C$ and $D$ were placed into 3D-bioreactors before been placed in the culture wells.

Fragments were cultured in a two-stage system: 14 days of standard media, followed by 6 or 8 days of activing A supplementation. The total culture time was therefore 20 or 22 days. The fragments were initially cultured for 14 days at $37^{\circ} \mathrm{C}$ in humidified air with $5 \% \mathrm{CO} 2$ with half the medium being changed every two days. This first stage of culture was set up because of the thickness of the ovarian fragments, to allow the atresia of the remaining antral follicles not visible under light microscopy. During the second culture stage, activin A was added in groups B and D. Previous studies in humans have tested an activing 
A supplementation duration of $2,4,7$ or 8 days $(9,13,14)$. With better results for the longest supplementations, we assessed two different supplementation conditions: 6 and 8 days.

\section{Histological evaluation}

Fresh and cultured ovarian tissues were fixed in paraformaldehyde 4\% (Sigma-Aldrich, Saint Louis, USA), dehydrated, included in paraffin, and cut into $3 \mu \mathrm{m}$ serial sections. Five sections were cut per ovarian fragment, equidistant from $60 \mu \mathrm{m}$ each, except for a $200 \mu \mathrm{m}$ gap between the $2^{\text {nd }}$ and the $3^{\text {rd }}$ section. The five sections per ovarian fragment were observed by light microscopy MOTIC Panthera (PantheraCC $\AA$, MoticEurope, Barcelone, Espagne) after being stained with hematoxylin and eosin (Sigma Alrich, Saint Louis, USA). A total of five fragments of fresh cortical tissue and 40 fragments of cultured tissue were fixed in paraformaldehyde $4 \%$ and processed for histological evaluation as described above. A total of 3519 follicles were examined under the light microscope.

\section{Follicular density}

The follicular density was compared between the fresh group and groups A, B, C and D after 20 or 22 days of culture. Follicles were classified according to their stage of development based on A. Gougeon classification as follows:

Primordial stage: oocyte surrounded by flattened granulosa cells,

Intermediate stage: oocyte surrounded by flattened and cuboidal granulosa cells,

Primary stage: oocyte surrounded only by cuboidal granulosa cells,

Secondary stage: oocyte surrounded by 2 or more layers of cuboidal granulosa cells,

Tertiary stage: antrum formation.

Follicular density of a tissue is defined as the number of follicles divided by the tissue surface. The integrated measuring tool in the ImageJ software (1.52 version) was used to measure fragments dimensions for follicle density.

\section{Follicular growth}

Secondary stage follicle, i.e. oocyte surrounded by 2 or more layers of cuboidal granulosa cells were measured with the integrated measuring tool in the Image $\mathrm{J}$ software. The mean follicle diameters were compared between the fresh group and groups A, B, C and D after 20 or 22 days of culture.

\section{Follicular quality and morphology}

The proportions of intact and atretic follicles were compared between the groups. The follicles were classified as follows: 
Intact follicle: intact follicle with a complete layer of granulosa cells and no alteration criteria

Altered follicle: empty follicle, cytoplasmic detachment, fushia staining, uncomplete layer of granulosa cells (figure 2).

\section{Detection of Ki67 and Cleaved Caspase 3 immunoreactivity in cultured follicles}

To evaluate follicular proliferation and apoptosis, Ki67 (9027; Cell Signaling Technology, Danvers, USA) and Cleaved Caspase 3 (9664S; Cell Signaling Technology) immunoreactivity were used. Slides in every culture group previously described were chosen. Only the slides with the greatest numbers of follicles seen in histology were selected to perform immunostaining. One slide contained two tissue sections; the upper one was used for Ki67 and the lower one for Cleaved Caspase 3. Each series had a negative and a positive control slide.

Ovarian tissue sections were dewaxed in methylcyclohexan (Sigma-Aldrich) and absolute ethanol (Sigma-Aldrich) then rinsed with water. The unmasking was done by an immersion of the slides in sodium citrate buffer ( $\mathrm{pH}=6$ (Merck, Darmstadt, Germany)) heated in a water bath during 45 minutes at $98^{\circ} \mathrm{C}$. The detection of the antigen was realized thanks to a kit based on peroxidase using $3,3^{\prime}-$

Diaminobenzidine (DAB) used as chromogen (EnVisionTM GI2 Doublestain System ${ }^{\circledR}$; Dako, Glostrup, Denmark). Tissue sections were incubated with Dual Endogenous Enzyme Block to block endogenous peroxidase then washed in Phosphate Buffered Saline (PBS). The sections were probed with the primary antibody during one hour at room temperature, washed with PBS then incubated with the Polymer/HRP reagent. DAB was finally applied for 15 minutes after a last washing with PBS. Counterstaining was achieved using standard hematoxylin staining (Millipore). Samples were mounted using an aqueous mounting medium (Dako Faramount ${ }^{\circledR} ;$ Dako). The two primary antibodies used were a monoclonal rabbit anti-Ki 67 antibody (9027; Cell Signaling Technology, Danvers, USA) which is a specific marker of cell proliferation with a dilution of $1 / 500$ and a monoclonal rabbit anti-cleaved caspase-3 antibody (9664S; Cell Signaling Technology) which is a specific marker of apoptotic cells with a dilution of $1 / 200$. Two readers independently observed the slides using light microscopy (Panthera $\mathrm{CC}^{\circledR}$; Motic Europe, Barcelona, Spain).

Tissue proliferation and apoptosis were expressed by a cell density. Marked cells with DAB chromogen were counted per tissue section surface. Follicle proliferation was assessed with a percentage of positive follicles over the total number of follicles. A follicle was considered as positive when at least one granulosa cell was stained. Regarding apoptosis, the follicle was considered in apoptosis if more than $50 \%$ of the cells were brown. In both cases, the oocyte was considered as a major cell, when positive the follicle was judged $100 \%$ in proliferation or apoptosis.

All the slides were read by two independent readers for histological evaluation, Ki67 and Cleaved Caspase 3 detection. 
Concentrations of estradiol in culture media were determined using a Chemiluminescent Microparticle Immunoassay CMIA (Abbott) at day 6, day 20 and day 22. The functional sensitivity of the estradiol assay was $<25 \mathrm{pg} / \mathrm{mL}$.

\section{Statistical analyzes}

Continuous variables are described with the mean and standard-deviation and categorical variables with the count and percentage. To manage the non-independence of the data, the effects of Activin A and 3Dstructure were analyzed using multivariate models considering the patients and the culture conditions (culture time and level). Follicular density, the proportion of marked follicles and the density of cells marked by immunostaining (Ki67 and Cleaved Caspase 3) were analyzed with a zero-inflated binomial negative model, suitable to over-dispersed counting data. The follicular diameter and estradiol level were analyzed with a linear regression model after logarithmic transformation. Due to non-homoscedastic residual variance, variance was modeled by considering the patient for the follicular diameter and the expected value for the estradiol level. A p-value less than 0.05 was considered significant. The analyzes were performed using R software, version 3.6.1 (15) with the "pscl" library (16).

\section{Results}

\section{Follicular density}

A decrease in follicular density was observed between the fresh and the cultured fragments $(3.8 \pm 2.0$ follicles $/ \mathrm{mm}^{2}$ vs $1.7 \pm 1.6$ and $2.1 \pm 1.8$ follicles $/ \mathrm{mm}^{2}$ (Days 20 and 22 ), $\mathrm{p}<0.001$ ). No significant difference was observed between the group with Activin compared to the group without $\left(1.9 \pm 1.6\right.$ follicles $/ \mathrm{mm}^{2} \mathrm{vs}$ $1.9 \pm 1.8$ follicles $\left./ \mathrm{mm}^{2}, \mathrm{p}>0.05\right)$. No significant difference was observed between the group with 3Dstructure compared to the group without ( $1.9 \pm 1.7$ follicles $/ \mathrm{mm}^{2}$ vs $1.9 \pm 1.7$ follicles $\left./ \mathrm{mm}^{2}, \mathrm{p}>0.05\right)$.

In freshly isolated uncultured tissue $62.4 \pm 27.35 \%$ of the follicles were identified as primordial. The proportion of primordial follicles in the cultured fragments were observed as follows: $15.4 \pm 4.6 \%$ at Day 20 and $14.7 \pm 4.5 \%$ at Day 22 in group $A ; 44.5 \pm 25.5 \%$ at Day 20 and $25.6 \pm 11.0 \%$ at Day 22 in group B; $24.8 \pm 13.3 \%$ at Day 20 and $0.9 \pm 0.3 \%$ at Day 22 in group C; and finally, $6.4 \pm 2.5 \%$ at Day 20 and $13.7 \pm 4.74 \%$ at Day 22 in group $D$. The proportion of primordial follicles was significantly reduced by culturing but not affected by Activin A supplementation or 3D-structure, $p<0.001$.

In freshly isolated uncultured tissue $20.2 \pm 2.96 \%$ of the follicles were identified as primary. The proportion of primary follicles in the cultured fragments were observed as follows: $30.5 \pm 11.7 \%$ at Day 20 and $39.6 \pm 12.6 \%$ at Day 22 in group $A ; 27.6 \pm 2.4 \%$ at Day 20 and $22.8 \pm 12.5 \%$ at Day 22 in group $B ; 21.3 \pm 8.4 \%$ at Day 20 and $56.8 \pm 39.2 \%$ at Day 22 in group C; and finally, $44.8 \pm 17.6 \%$ at Day 20 and $58.9 \pm 34.4 \%$ at Day 22 in group D. The proportion of primary follicles was significantly increased by culturing, $p<0.001$.

In freshly isolated uncultured tissue $6.9 \pm 1.0 \%$ of the follicles were identified as secondary. The proportion of secondary follicles in the cultured fragments were observed as follows: $29.8 \pm 19.6 \%$ at Day 20 and 
$18.9 \pm 10.2 \%$ at Day 22 in group $A ; 27.6 \pm 13.5 \%$ at Day 20 and $15.3 \pm 5.7 \%$ at Day 22 in group $B ; 12.0 \pm 5.7 \%$ at Day 20 and $31.4 \pm 24.2 \%$ at Day 22 in group C; and finally, $25.7 \pm 17.4 \%$ at Day 20 and $13.3 \pm 7.4 \%$ at Day 22 in group $D$. The proportion of secondary follicles was significantly increased by culturing, $p<0.001$.

Antral cavities were observed in the 4 cultured groups.

Activin A supplementation did not significantly affect the follicular distribution between primordial, intermediate, primary, and secondary follicles, $p>0.05$. However, the 3D-structure increased the density of primary follicles only (RR=1.39, IC95\%: 1.14-1.70, $p<0.001$ ) (Figures 3 and 5).

\section{Follicular growth}

Follicles were cultured for 20 or 22 days in 4 culture groups. No significant follicle diameter growth was observed between Day $0(70.0 \mu \mathrm{m} \pm 19.2)$ and the four groups at Day $20(63.6 \mu \mathrm{m} \pm 18.9)$ or the four groups at Day $22(70.4 \mu \mathrm{m} \pm 28.4)$.

Between day 20 and day 22, the diameters of follicles cultured without a 3D-structure were greater $(69.6 \pm 21.2 \mu \mathrm{m})$ than those cultured within a three-dimensional structure $(63.5 \mu \mathrm{m} \pm 27.2)(p<0.001)$. Activin A supplementation did not significantly influence the mean diameter of follicles $(65.7 \mu \mathrm{m} \pm 29.1$ with Activin A vs $68.0 \mu \mathrm{m} \pm 18.0$ without, $p>0.05$ ).

\section{Follicular quality and morphology}

The mean of intact follicles in the cultured fragments was observed as follows: $42.7 \pm 34.7 \%$ at Day 20 and $54.3 \pm 33.6 \%$ at Day 22 in group $A ; 27.7 \pm 16.4 \%$ at Day 20 and $35.7 \pm 33.0 \%$ at Day 22 in group $B$; $17.5 \pm 29.1 \%$ at Day 20 and $62.9 \pm 32.7 \%$ at Day 22 in group C; and finally, $54.5 \pm 5.0 \%$ at Day 20 and $54.1 \pm 31.4 \%$ at Day 22 in group D. Qualitative assessment indicated no difference between the groups, $p>0.05$. The presence of Activin A or 3D-structure did not significantly affect follicular quality, $p>0.05$.

\section{Estradiol secretion}

Media from ovarian fragments cultured individually were analyzed for estradiol content by chemiluminescent microparticle immunoassay (CMIA). In group A, the estradiol levels were $120.00 \pm$ $55.43 \mathrm{pg} / \mathrm{ml}$ at D6, $5151.00 \pm 3463.23 \mathrm{pg} / \mathrm{ml}$ at D20 and $3323.60 \pm 3780.42 \mathrm{pg} / \mathrm{ml}$ at D22. In group B, estradiol levels were $146.00 \pm 10.39 \mathrm{pg} / \mathrm{ml}$ at D6, $2227.20 \pm 1736.04 \mathrm{pg} / \mathrm{ml}$ at D20 and $1738.60 \pm$ $1002.98 \mathrm{pg} / \mathrm{ml}$ at D22. In group C, estradiol levels were $237.33 \pm 162.24 \mathrm{pg} / \mathrm{ml}$ at $\mathrm{D} 6,446.80 \pm$ $326.12 \mathrm{pg} / \mathrm{ml}$ at $\mathrm{D} 20$ and $1130.20 \pm 683.07 \mathrm{pg} / \mathrm{ml}$ at D22. In group $\mathrm{D}$, estradiol levels were $680.33 \pm$ $551.95 \mathrm{pg} / \mathrm{ml}$ at D6, $854.20 \pm 990.17 \mathrm{pg} / \mathrm{ml}$ at D20 and $1965.00 \pm 1650.00 \mathrm{pg} / \mathrm{ml}$ at D22. Significant estradiol secretion was observed in cultured ovarian fragments at days 20 and 22 compared with the day 6 group ( $p<0.001$ ) (Figure 4). Estradiol levels were estimated to be $1795.6 \pm 2680.7 \mathrm{pg} / \mathrm{ml}$ in the activin $A$ supplemented groups versus $1287.5 \pm 1170.5 \mathrm{pg} / \mathrm{ml}$ in the non-activin A groups. There was no statistically significant difference between the groups ( $R R=1.33, C 195 \%: 0.91-1.94, p>0,05)$. The presence of activin A did not significantly alter the estradiol level of the culture medium. Significant estradiol 
secretion was observed in ovarian fragments cultured without 3D-structure. Estradiol levels were measured at $802,8 \pm 904,3 \mathrm{pg} / \mathrm{ml}$ in the presence of the $3 \mathrm{D}$-structure and $2280,3 \pm 2597,9 \mathrm{pg} / \mathrm{ml}$ without. A decrease of 2.5 in estradiol levels was observed in the presence of the 3D-structure $(p<0.001)$

\section{Detection of Ki67 antigen}

Sixty slides with 60 tissue sections were evaluated for Ki67, equally distributed in each four groups. Tissue and follicle proliferation were confirmed by the detection of the proliferating nuclear antigen Ki67 on several growing follicles and tissue cells from the control slides. The mean cellular density of stromal cells within the ovarian tissue was significantly less important in the group with 3D-structure than in the group without $\left(7.1 \pm 12.3\right.$ cells $/ \mathrm{mm}^{3}$ vs $14.5 \pm 21.7$ cells $\left./ \mathrm{mm}^{3}, \mathrm{p}=0.008\right)$. The difference between the group with Activin compared to the group without was not significant $\left(7.4 \pm 12.1 \mathrm{cells} / \mathrm{mm}^{3} \mathrm{vs} 14.1 \pm 22.0\right.$ cells $\left./ \mathrm{mm}^{3}, \mathrm{p}=0.544\right)$.

The difference regarding the mean number of positive follicles for proliferation was not significant within the group with the 3D-structure compared to the group without ( $3.2 \pm 8.6 \%$ vs $14.9 \pm 26.6 \%, p=0.081$ ) and in the group with Activin A compared to the group without ( $5 \pm 17.1 \%$ vs $12.8 \pm 22.5 \%, p=0.108$ ).

\section{Detection of Cleaved Caspase 3 antigen}

The same slides were used to evaluate the presence of Cleaved Caspase 3.

Tissue and follicle apoptosis were confirmed by the detection of the apoptosis nuclear antigen Cleaved Caspase 3 on several wasted follicles and tissue cells from the control slides.

The difference in cellular density within the ovarian tissue was not significant between the group with the 3D-structure compared to the group without ( $5.1 \pm 5.3$ cells $/ \mathrm{mm} 3$ vs $9.6 \pm 14.8$ cells $/ \mathrm{mm} 3, p=0.057$ ). Although, it was significantly more important in the group with Activin A compared to the group without (10.4 \pm 14 cells $/ \mathrm{mm} 3$ vs $3.5 \pm 2.9$ cells $/ \mathrm{mm} 3, \mathrm{p}=0.006)$.

The difference regarding the mean number of positive follicles for apoptosis was not significant between the group with the 3D-structure compared to the group without (10.1 $\pm 14.8 \%$ vs $14.5 \pm 24.3 \%, p=0.318)$ and in the group with Activin A compared to the group without (13.8 $\pm 22.7 \%$ vs $10.3 \pm 15.7 \%, p=0.859)$.

\section{Discussion}

The objective of this study was to evaluate a new 3D-structure with or without Activin A on the activation of folliculogenesis in human ovaries.

We found in this study a decrease in follicular density between the fresh and the cultured fragments. Our results were in accordance with the literature and have already been described in the study of Ding et al. (13). It may be explained by an alteration of the tissue after the surgery. Even though the time and transit of the ovaries were optimized, the interruption of the vascularization is probably responsible for some 
tissue damage. Simultaneously, spontaneous folliculogenesis was observed in all four groups regardless of the presence of the 3D-structure or Activin A. The repressive action of ovarian tissue on folliculogenesis in the human body through inhibitory factors has already been described $(9,17,18,19)$. Moreover, it is already well known that ovarian fragmentation, by disrupting the Hippo signaling pathway, induces follicular growth (20). In all four groups we found a decrease in primordial follicles and an increase in primary and secondary follicles. These results are aligned with other studies on the subject (2, $9,13)$. Nonetheless, it must be underlined that we found a more important density of primary follicles with the structure than without, which has never been described. Moreover, we found tertiary follicles in all groups. Many authors found a positive impact of Activin A for the development of tertiary follicles $(2,9$, 13). We did not find this effect in our study.

Regarding the mean follicular diameter, it was significantly smaller in the 3D-structure group than in the group without any structure. This was already described in the Higuchi et al. study with the use of Matrigel ${ }^{\circledR}(21)$. Two hypotheses may explain this difference. The first one is the necrosis of the follicles creating a swelling and a disruption of granulosa cells leading to an increase of their size before the scattering of the cytoplasmic and nuclear contents. The second one could be the disjunction of the tissue cultivated without any structure. The expression of connexin 43 was already described in two studies (2, 22) showing an increase of this protein in the group with Activin A. We did not find an impact of Activin A on follicular diameters.

Regarding follicular quality, no significant difference was found between the four groups. Ding et al. and Telfer et al. did not find a positive impact of Activin A on follicular quality whereas it was described in mouse and sheep $(9,13,23,24)$. It must be mentioned that even though quality and follicular morphology were evaluated by two readers, human assessment always remains subjective.

Concerning the assessment of the steroidogenesis function of the ovary we measured the estradiol level in culture media. We observed an increase of the estradiol level in all groups proving the differentiation and functionality of the granulosa cells. It was already described in the literature (9). More importantly, we found a higher level in both groups without any structure. Here again, two hypotheses are raised to explain that estradiol level is less important in the 3D-structure. The first one could be the releasing of estradiol after cellular lysis. The second one could be a pejorative uproar of the folliculogenesis with an increase of estradiol synthesis.

Regarding proliferation and apoptosis, we found more Ki67 and Cleaved Caspase 3 stained cells in both groups without 3D-structure. This supports our hypothesis of a pejorative too fast folliculogenesis activation without a structure. Indeed, the more apoptosis is found the more proliferation is present. Regarding Activin A, we found the same phenomenon in the group without Activin A. On the contrary some authors found more proliferation with Activin A; apoptosis was not studied as we did. Here again the results on Activin A remains unclear.

Some limitations must be underlined in our study. The first one is the potential presence of secondary follicles at the beginning of the culture which may have evolved into antral follicles. Those follicles could 
have been counted as new ones whereas they were already there. We tried to remove them when they were at the antral stage. However, it is very likely that some of them at the secondary stage remained. Then, the small antral follicles produce $\mathrm{AMH}$ and could have inhibited the recruitment of primordial follicles. In the study of Mc Laughlin et al, they removed the follicles with a diameter superior to $40 \mu \mathrm{m}$ (2). Nonetheless, as in Ding et al, Higuchi et al, Cossigny et al and da Silva et al we always compared our cultured tissue to the fresh one $(13,21,23,24)$.

Secondly, human ovaries were removed from patients who had testosterone before the surgery for a duration varying between three months to three years. This could have had an impact on the ovarian environment. However, in a recent oral conference, E Telfer, obtained less follicles and a slower follicular growth in tissue exposed to male hormones than in non-exposed tissue (IVF worldwide, 2020). Then another main limitation was the heterogeneity of the ovarian tissue concerning the ovarian reserve. As a matter of fact, a tissue with more follicles at baseline may be easier to activate than another tissue with a poor follicular reserve.

To finish with, only five tissues were used. It could appear to be a small number of samples. Nevertheless, compared to other articles on in vitro folliculogenesis it is already substantial. As a matter of fact, we look at 3499 follicles whereas in other studies the numbers of follicles studied were between 74 and 1137 .

To conclude, our study found a spontaneous in vitro folliculogenesis activation after culture despite the culture system used. We did not find a real benefit of using Activin A which is quite different from other works on this subject. Notwithstanding, our study suggests that our 3D-structure may prevent deleterious, excessive, and too fast activation of folliculogenesis. In vivo folliculogenesis is a really long process, creating a good follicle with a good oocyte needs time. To date only two teams, succeed in producing a mature follicle in humans $(2,26)$. Our next step will be to cultivate ovarian fragments longer (during many months) with or without the structure and add Activin A thereafter. Another ovarian model is also needed. The purpose of all this research is to be able one day to have another viable and better option for fertility preservation.

\section{Conclusion}

Activin A did not have any impact on follicular growth at the beginning of the culture. Our new bioreactor seemed to allow a more physiological in vitro folliculogenesis activation.

\section{Declarations}

Ethics approval and consent to participate: A written consent form was obtained for all patients and the institutional review board approved the study.

Consent for publication: Not applicable. 
Availability of data and materials: The dataset used and/or analyzed during the current study are available from the corresponding author on a reasonable request.

Competing interests: The authors declare that they have no competing interests.

Funding: None.

Authors' contribution: EL designed the study. NMJ performed the tissue extraction. CJ, EF, and CF performed all the preparations of the tissue. AG made the statistics. CJ, EF, and EL analyzed and interpreted the results. LD, EL and AM designed the bioreactor. $\mathrm{CJ}$ and $\mathrm{EF}$ wrote the manuscript and $\mathrm{EL}$ corrected it. All the authors read and approved the final manuscript.

Acknowledgments: None.

\section{References}

1. - Lotz L, Dittrich R, Hoffmann I, et al. Ovarian Tissue Transplantation: Experience from Germany and Worldwide Efficacy. Clin Med Insights Reprod Health. 2019;13:1-8.

2. - McLaughlin M, Bromfield JJ, Albertini DF, et al. Activin promotes follicular integrity and oogenesis in cultured pre-antral bovine follicles. Mol Hum Reprod. 2010 Sep;16(9):644-53.

3. - Brevini TAL, Pennarossa G, Gandolfi F. A 3D approach to reproduction. Theriogenology. 2020 Jan 11.

4. - Sadr SZ, Fatehi R, Maroufizadeh S, et al. Utilizing Fibrin-Alginate and Matrigel-Alginate for Mouse Follicle Development in Three-Dimensional Culture Systems. Biopreservation Biobanking. 2018;Apr;16(2):120-7.

5. - Joo S, Oh S-H, Sittadjody S, et al. The effect of collagen hydrogel on 3D culture of ovarian follicles. Biomed Mater Bristol Engl. 2016;11(6):065009. 11(.

6. - Zuccotti M, Merico V, Rebuzzini P, et al. 3D culture of ovarian follicles: a system towards their engineering? Int J Dev Biol. 2015;59(4-6):211-6.

7. - Simon LE, Kumar TR, Duncan FE. In vitro ovarian follicle growth: a comprehensive analysis of key protocole variables. Biol Reprod. 2020;103(3):455-70.

8. - Bloise E, Ciarmela P, Dela Cruz C, et al. Activin A in Mammalian Physiology. Physiol Rev. 2019;01(1):739-80. 99(.

9. - Telfer EE, McLaughlin M, Ding C, et al. A two-step serum-freeculture system supports development of human oocytes from primordial follicles in the presence of activin. Hum Reprod Oxf Engl. 2008 May; 23(5):1151-8.

10. - David L, Domard A, Rivas-Araiza R, Hollow. notably multi-membrane fibers, method for preparation thereof by spinning and device for applying Said method. Patent: US; 2008. p. 2012040463.

11. - Araiza RNR, Rochas C, David L, Domard A Interrupted Wet-Spinning Process for Chitosan Hollow Fiber Elaboration. Macromol. Symp. 2008, 266: 1-5. 
12. - Montembault A, Viton C, Domard A. Physico-chemical studies of the gelation of chitosan in a hydroalcoholic medium. Biomaterials 2005, 26: 933-43.

13. - Ding CC, Thong KJ, Krishna A, et al. Activin A inhibits activation of human primordial follicles in vitro. J Assist Reprod Genet. 2010 Apr;27(4):141-7.

14. - McLaughlin M, Albertini DF, Wallace WHB, et al. Metaphase II oocytes from human unilaminar follicles grown in a multi-step culture system. Mol Hum Reprod. 2018;01(3):135-42. 24(.

15. - R Core Team. R: A language and environment for statistical computing. Vienna: R Foundation for Statistical Computing; 2019.

16. Zeileis -A, Kleiber C, Simon Jackman 2008, Regression Models for Count Data in R. Journal of Statistical Software 27.

17. - Fortune JE, Cushman RA, Wahl CM, et al. The primordial to primary follicle transition. Mol Cell Endocrinol. 2000;63(1-2):53-60.

18. - Wandji SA, Srsen V, Voss AK, et al. Initiation in vitro of growth of bovine primordial follicles. Biol Reprod. 1996;55(5):942-8.

19. - Hovatta O, Silye R, Abir R, et al. Extracellular matrix improves survival of both stored and fresh human primordial and primary ovarian follicles in long-term culture. Hum Reprod. 1997;12:1032-6.

20. - Kawamura K, Chen Y, Suzuki N, et al. Hippo signaling disruption and Akt stimulation of ovarian follicles for infertility treatment. PNAS. 2013;110(43):17474-9.

21. - Higuchi CM, Maeda Y, Horiuchi T, et al. A Simplified Method for Three-Dimensional (3-D) Ovarian Tissue Culture Yielding Oocytes Competent to Produce Full-Term Offspring in Mice. PloS One. 2015;10(11):e0143114.

22. - Nagashima JB, Wildt DE, Travis AJ, et al. Activin promotes growth and antral cavity expansion in the dog ovarian follicle. Theriogenology. 2019 Apr;129:168-77.

23. - Cossigny DA, Findlay JK, Drummond AE. The effects of FSH and activin A on follicle development in vitro. Reprod Camb Engl. 2012 Feb;143(2):221-9.

24. - Martins da Silva SJ, Bayne RL, Cambray N, Hartley PS, McNeilly AS, Anderson RA. Expression of activin subunits and receptors in the developing human ovary: activin A promotes germ cell survival and proliferation before primordial follicle formation. Dev Biol. 2004 Feb 15; 266(2):334-45.

25. - Wongbandue G, Jewgenow K, Chatdarong K. Effects of thyroxin (T4) and activin A on in vitro growth of preantral follicles in domestic cats. Theriogenology. 2013 Mar;79(5):824-32.

26. - Xu F, Lawson MS, Bean Y, et al. Matrix-free 3D culture supports human follicular development from the unilaminar to the antral stage in vitro yielding morphologically normal metaphase II oocytes. Hum Reprod. 2021 Apr 20; 36(5): 1336-38.

\section{Figures}




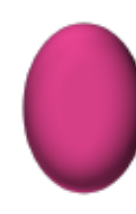

Fresh ovary

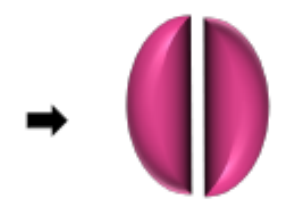

2 hemi cortexes

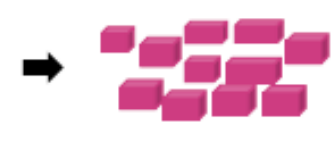

Ovarian fragments
$\Rightarrow \quad 5$ ovarian tissues

- Fresh tissue assessment $(n=5)$

- Tissue culture : 4 groups $(n=40)$

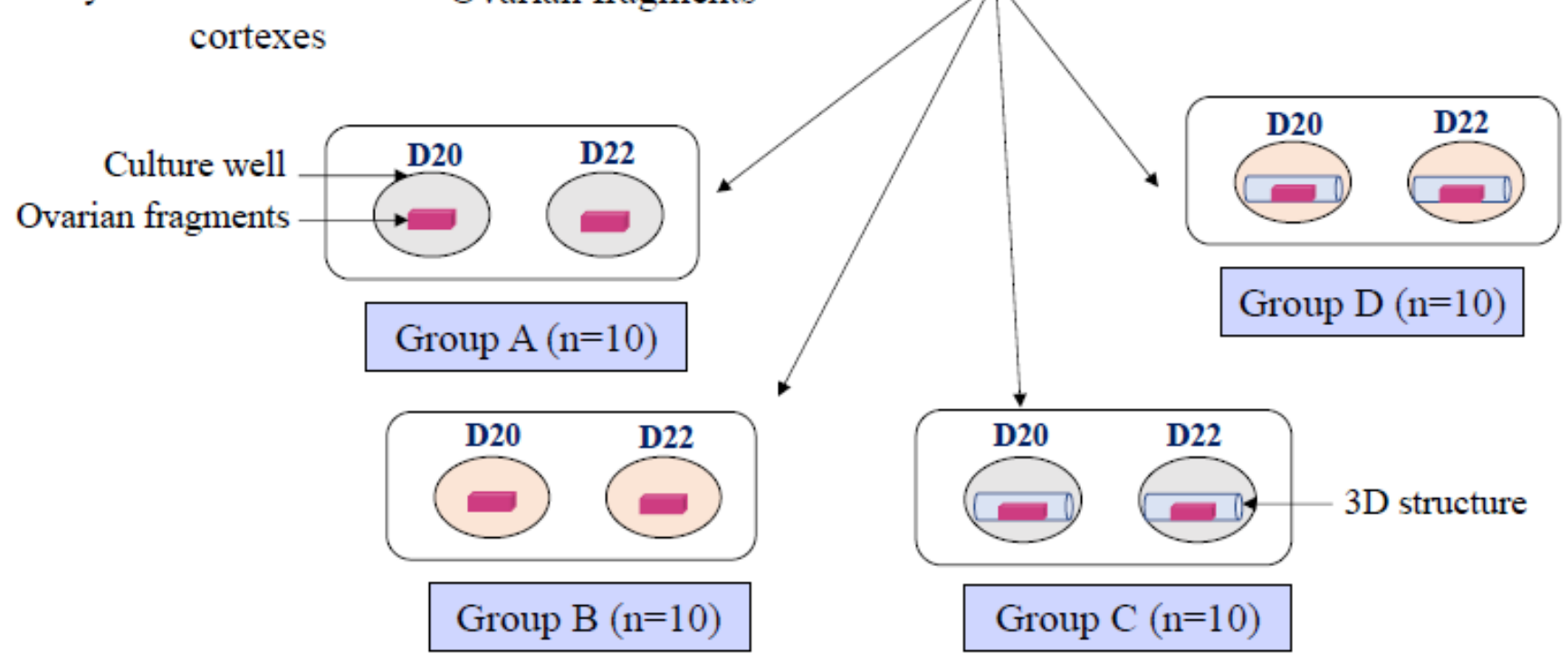

\section{Figure 1}

Design of the work. The fresh ovary was cut lengthwise in two fragments. For each fragment, the medulla was removed, thus obtaining two hemi cortexes. These hemi cortexes were dissected into ovarian fragments. One fresh fragment was taken for fresh tissue assessment whereas the remaining fragments were placed randomly in four culture groups for 20 or 22 days : - Group A : Ovarian fragment placed in culture medium without Activin A without 3D-structure - Group B : Ovarian fragment placed in culture medium with Activin A but without 3D-structure - Group C : Ovarian fragment placed in culture medium without Activin A but within a 3D-structure - Group D : Ovarian fragment placed in culture medium with Activin A within 3D-structure 


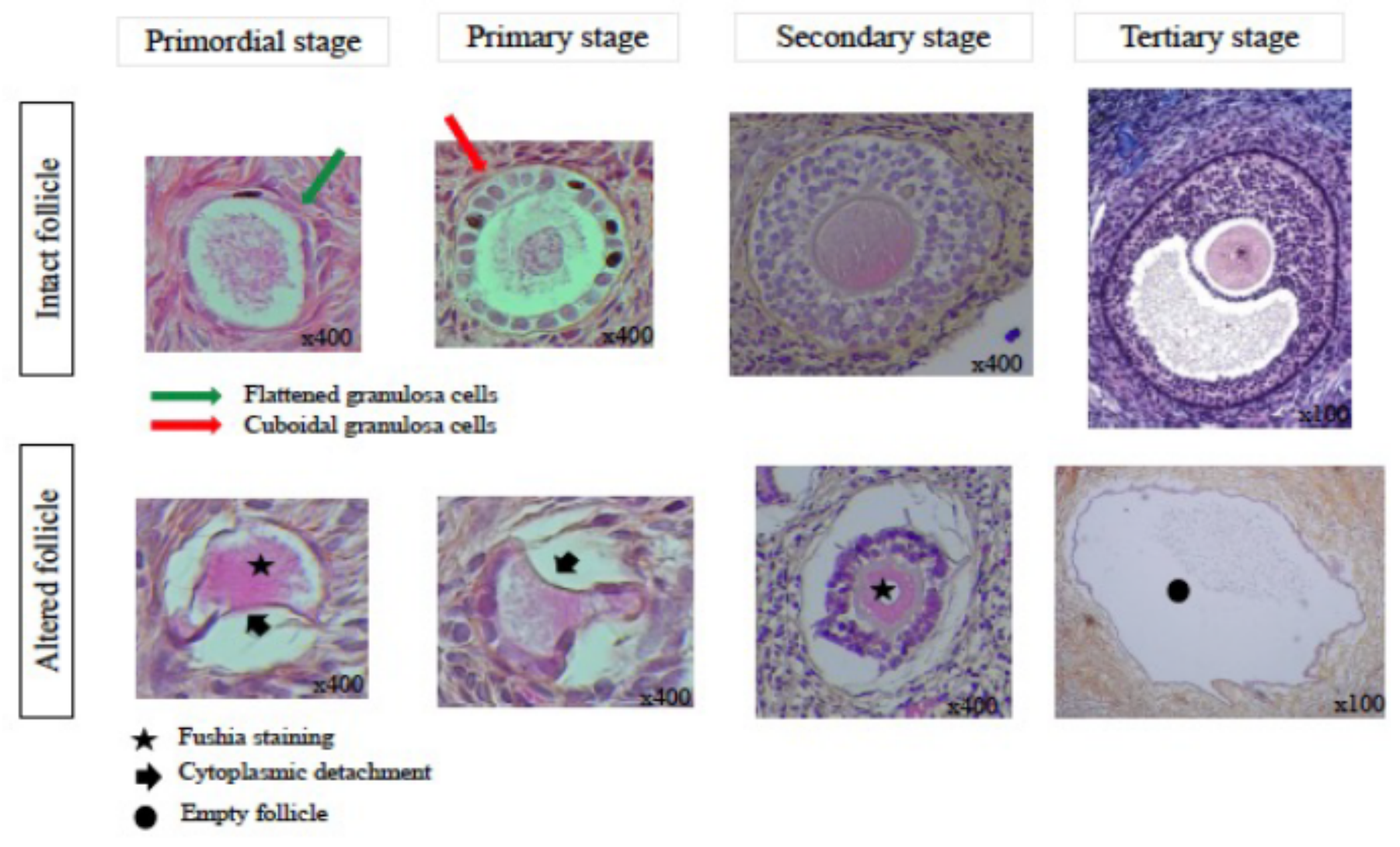

Figure 2

Classification according to follicle morphology: intact tertiary follicle (A. Gougeon) altered tertiary follicle (personal pictures) x 100; all other follicles (personal picture) x 400

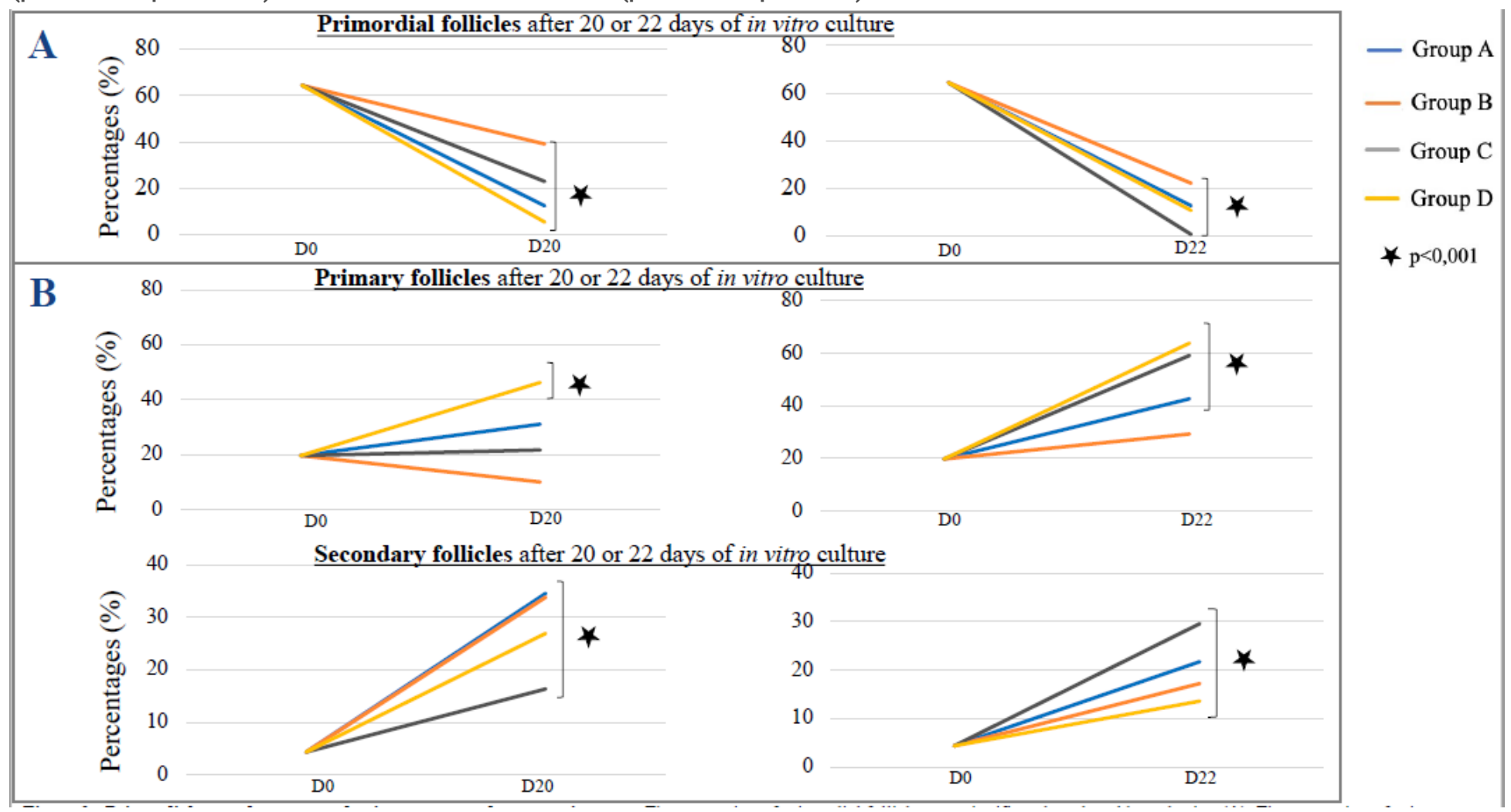




\section{Figure 3}

Primordial stage decrease, and primary - secondary stage increase. The proportion of primordial follicles was significantly reduced by culturing $(A)$. The proportion of primary and secondary follicles was significantly increased by culturing (B).

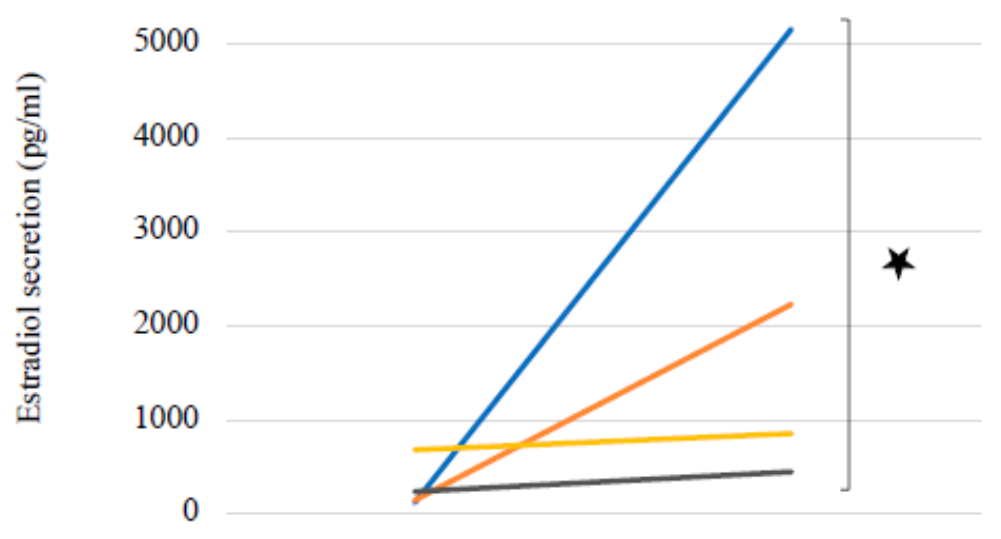

D6

D20

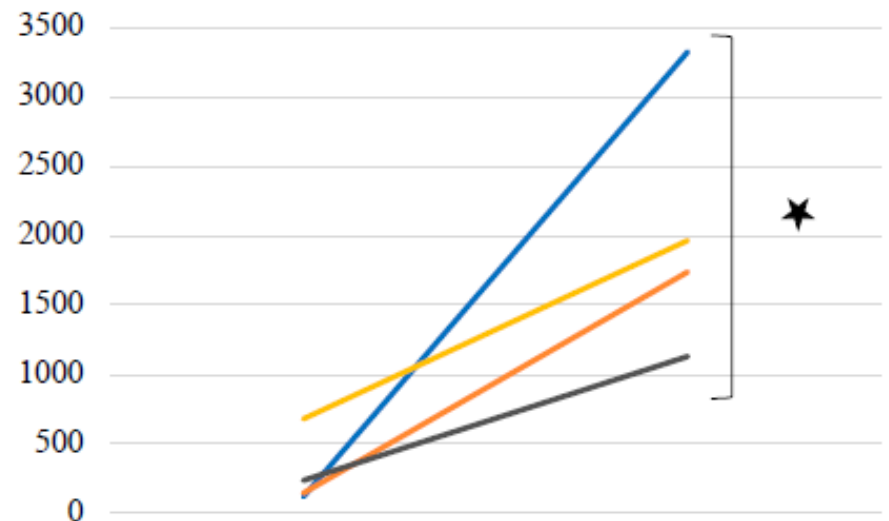

D6

D22

- Group A - Group B - Group C - Group D * $\quad$ p $<0,001$

\section{Figure 4}

Estradiol secretion after 6, 20 and 22 days of in vitro culture. Significant estradiol secretion was observed in ovarian fragments at days 20 and 22 compared with day 6 group. A decrease of 2,5 in estradiol was observed in the presence of the 3D-structure.

\begin{tabular}{|c|c|c|c|c|c|c|c|c|c|c|c|c|c|}
\hline \multirow{2}{*}{\multicolumn{2}{|c|}{ Group }} & & \multirow{2}{*}{ Total } & \multicolumn{2}{|c|}{ Primordial stage } & \multicolumn{2}{|c|}{ Intermediate stage } & \multicolumn{2}{|c|}{ Primary stage } & \multicolumn{2}{|c|}{ Secondary stage } & \multicolumn{2}{|c|}{ Tertiary stage } \\
\hline & & & & Intact & Altered & Intact & Altered & Intact & Altered & Intact & Altered & Intact & Altered \\
\hline \multirow{2}{*}{\multicolumn{2}{|c|}{ Fresh }} & Total number of follicles & 637 & 22 & 375 & 5 & 61 & 27 & 102 & 17 & 27 & 0 & 1 \\
\hline & & Follicular stage by group (\%) & & 3.5 & 58.9 & 0.8 & 9.6 & 4.2 & 16.0 & 2.7 & 4.2 & 0 & 0.2 \\
\hline \multirow{8}{*}{ D20 } & \multirow{2}{*}{ A } & Total number of follicles & 265 & 3 & 38 & 4 & 59 & 25 & 56 & 61 & 18 & 1 & 0 \\
\hline & & Follicular stage by group (\%) & & 1.1 & 14.3 & 1.5 & 22.2 & 9.4 & 21.1 & 23.0 & 6.8 & 0.4 & 0 \\
\hline & \multirow{2}{*}{ B } & \multirow{2}{*}{$\begin{array}{l}\text { Total number of follicles } \\
\text { Follicular stage by group (\%) }\end{array}$} & 301 & 16 & 118 & 10 & 41 & 12 & 17 & 37 & 46 & 1 & 3 \\
\hline & & & & 5.3 & 39.2 & 3.3 & 13.6 & 4.0 & 5.6 & 12.3 & 15.2 & 0.3 & 1.0 \\
\hline & \multirow{2}{*}{ C } & \multirow{2}{*}{$\begin{array}{l}\text { Total number of follicles } \\
\text { Follicular stage by group (\%) }\end{array}$} & 423 & 2 & 103 & 1 & 172 & 24 & 66 & 28 & 23 & 2 & 2 \\
\hline & & & & 0.5 & 24.3 & 0.2 & 40.7 & 5.7 & 15.6 & 6.6 & 5.4 & 0.5 & 0.5 \\
\hline & \multirow{2}{*}{ D } & \multirow{2}{*}{\begin{tabular}{|l|} 
Total number of follicles \\
Follicular stage by group (\%)
\end{tabular}} & 315 & 11 & 9 & 33 & 36 & 85 & 56 & 47 & 34 & 2 & 2 \\
\hline & & & & 3.5 & 2.9 & 10.5 & 11.4 & 27.0 & 17.8 & 14.9 & 10.8 & 0.6 & 0.6 \\
\hline \multirow{8}{*}{ D22 } & \multirow{2}{*}{ A } & \multirow{2}{*}{$\begin{array}{l}\text { Total number of follicles } \\
\text { Follicular stage by group (\%) }\end{array}$} & 535 & 13 & 66 & 35 & 107 & 123 & 89 & 84 & 17 & 1 & 0 \\
\hline & & & & 2.4 & 12.3 & 6.5 & 20.0 & 23.0 & 16.6 & 15.7 & 3.2 & 0.2 & 0 \\
\hline & \multirow{2}{*}{ B } & \multirow{2}{*}{\begin{tabular}{|l|} 
Total number of follicles \\
Follicular stage by group (\%)
\end{tabular}} & 347 & 5 & 85 & 14 & 109 & 50 & 29 & 33 & 20 & 1 & 1 \\
\hline & & & & 1.4 & 24.5 & 4.0 & 31.4 & 14.4 & 8.4 & 9.5 & 5.8 & 0.3 & 0.3 \\
\hline & \multirow{2}{*}{ C } & \multirow{2}{*}{\begin{tabular}{|l|} 
Total number of follicles \\
Follicular stage by group (\%)
\end{tabular}} & 229 & 0 & 2 & 10 & 14 & 71 & 59 & 52 & 20 & 0 & 1 \\
\hline & & & & 0.0 & 0.9 & 4.4 & 6.1 & 31.0 & 25.8 & 22.7 & 8.7 & 0 & 0.4 \\
\hline & \multirow{2}{*}{ D } & \multirow{2}{*}{$\begin{array}{l}\text { Total number of follicles } \\
\text { Follicular stage by group (\%) }\end{array}$} & 467 & 5 & 59 & 21 & 42 & 150 & 125 & 49 & 13 & 1 & 2 \\
\hline & & & & 1.1 & 12.6 & 4.5 & 9.0 & 32.1 & 26.8 & 10.5 & 2.8 & 0.2 & 0.4 \\
\hline
\end{tabular}

\section{Figure 5}


Total number of follicles counted for primordial, intermediate, primary, secondary and tertiary stage in fresh and after culture Fresh : One fresh fragment per patient was evaluated before tissue culture D20 : The fragments were cultured for 20 days at $37^{\circ} \mathrm{C}$ in humidifed air with 5\% C02 D22 : The fragments were cultured for 22 days at $37^{\circ} \mathrm{C}$ in humidifed air with $5 \% \mathrm{C0} 2$ Group A : Ovarian fragment placed in culture medium without Activin A without 3D-structure Group B : Ovarian fragment placed in culture medium with Activin A but without 3D-structure Group C : Ovarian fragment placed in culture medium without Activin $A$ but within a 3D-structure Group D : Ovarian fragment placed in culture medium with Activin A within 3Dstructure 